\title{
The Application of COMSOL Secondary Development Technology in the Fluid-solid Coupling Study of Prionace Glauca Pectoral Fins
}

\author{
Qing-Song HU ${ }^{1, a}$, Xi-Wu LIU ${ }^{1}$ and Wen-Ping CHENG ${ }^{1}$ \\ ${ }^{1}$ College of Engineering, Shanghai Ocean University, Shanghai, 201306, China
}

\author{
Keywords: Prionace glauca, Pectoral fins, COMSOL, Secondary development, Fluid-solid \\ coupling.
}

\begin{abstract}
During the cruise process of prionace glauca, its pectoral fins play an important role in keeping balance and stability of its body. The research on the fluid-solid coupling of prionace glauca pectoral fins is of important value to optimizing the design of the AUV and other marine engineering equipment. This paper studies the deformation shrinkage process of coupling between prionace glauca pectoral fins and the flow through the physical field technology of COMSOL fluid-solid coupling, and carries out the secondary development of COMSOL simulation interface to create App, and builds an intuitive user interface which is suitable for the fluid-solid coupling research of prionace glauca pectoral fins. The research result can improve the ability of modelling and post-processing, avoid the user input errors and focus on the output at the same time. The secondary development in this paper provides an important reference for COMSOL in practical engineering application.
\end{abstract}

\section{Introduction}

Prionace glauca pectoral fins play an important role in keeping balance and stability of its body, and has the characteristics of stable swimming posture, high efficiency and long-distance cruising $[1,2]$. One of the deficiencies of Autonomous Underwater Vehicle (AUV) is the poor energy efficiency and the low-level hydrodynamic performance. The research on the fluid-solid coupling of prionace glauca pectoral fins will contribute to solve the problem. Based on COMSOL Multiphysics Software, this paper studies the deformation mechanism of the prionace glauca pectoral fin under the special elastic structure. COMSOL Multiphysics has powerful multiphysics coupled computing capability with high accuracy and fast computations. But for the complex model, its pre-treatment, parameter setting and changing process is more complex, so the targeted secondary development of COMSOL Multiphysics is particularly important.

In recent years, the secondary development of COMSOL Multiphysics plays an important role in the actual engineering calculation. Huang[3] prepares the M-file script file to solve the seepage problem through the secondary development tools of the COMSOL Multiphysics, achieving the goal of seamless docking between the finite element analysis and optimization of Matlab. As for the specific problems of coal and gas outburst, Wei [4] directly defines the physical parameters and creates the appropriate model by using existing equations of COMSOL Multiphysics, inputting his own equations and a number of professional modules of the software, and utilizing the secondary development environment of the software to re-adjust and control the output of model calculation. $\mathrm{Li}[5]$ makes the secondary development on the finite element numerical calculation platform of COMSOL to accomplish the simulation process of three-dimensional aeration repairing . Wang [6] develops multi-physics-wide coupling analysis model and the computing procedures through $\mathrm{M}$ language of COMSOL Multiphysics platform.

According to the above researches, the secondary development of COMSOL Multiphysics is helpful to improve the accuracy of multi-physics coupling calculation, simplify the complex operation and improve the calculation efficiency. This paper develops a simulation App for the secondary development of COMSOL software and creates an intuitive and user-friendly interface for the study of fluid-solid coupling of prionace glauca pectoral fins, which improves the ability of 
modelling and post-processing. The simulation App collects all the parameters that influence the calculation of the fluid-solid coupling of the prionace glauca pectoral fin, so that those who do not have the simulation expertise can set their own numerical tests and enhance their understanding of the fluid-solid coupling simulation of the prionace glauca pectoral fins.

\section{Fluid-solid Coupling Model}

\section{Building Model}

This paper aims to obtain the bidirectional coupling mechanical features of prionace glauca pectoral fins in the flow field, and the prionace glauca pectoral fins play an important role in keeping balance, so it needs to focus on describing the shape characteristics of prionace glauca pectoral fins.The main characteristics of prionace glauca are as follows: the total length is $2131.0 \mathrm{~mm}$, maximum width $1102.2 \mathrm{~mm}$, tail length $410.0 \mathrm{~mm}$, maximum height $558.5 \mathrm{~mm}$, pectoral fin length $440.6 \mathrm{~mm}$, the maximum width of pectoral fin $190.5 \mathrm{~mm}$, the horizontal abduction angle 5 degree. The research builds the 3D model of prionace glauca based on the above dimension of prionace glauca shape. According to the need of calculation, rectangular region is selected as the calculation domain. As shown in Figure.1, the height is $1500 \mathrm{~mm}$, width is $2000 \mathrm{~mm}$ and length is $3000 \mathrm{~mm}$.

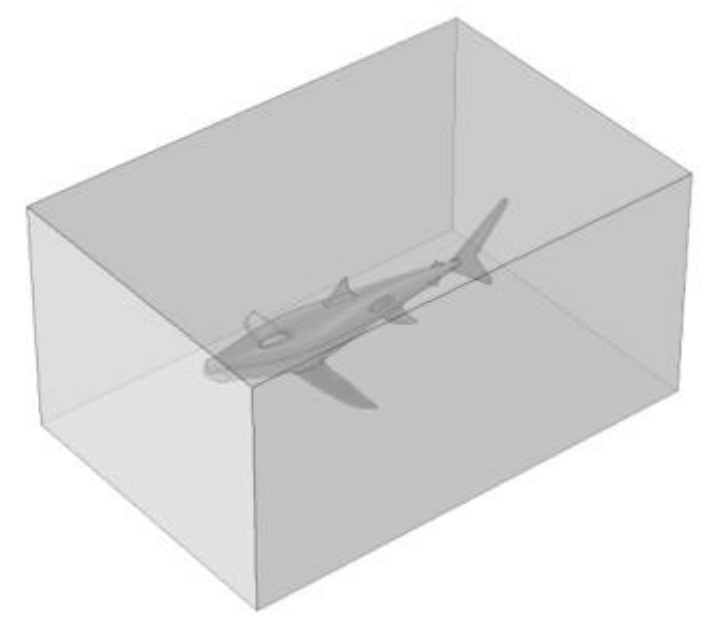

Fig.1.The calculation domain model

\section{Meshing}

This paper uses the finite element method to analyze fluid-structure interaction, so reasonable meshing is crucial.

The entire calculation area is divided into tetrahedral meshes, and then refines the mesh of key description parts such as the connection between fins and the trunk. The above works enhance the accuracy of the simulation results. Figure 2 shows the meshing results of the computational domain.

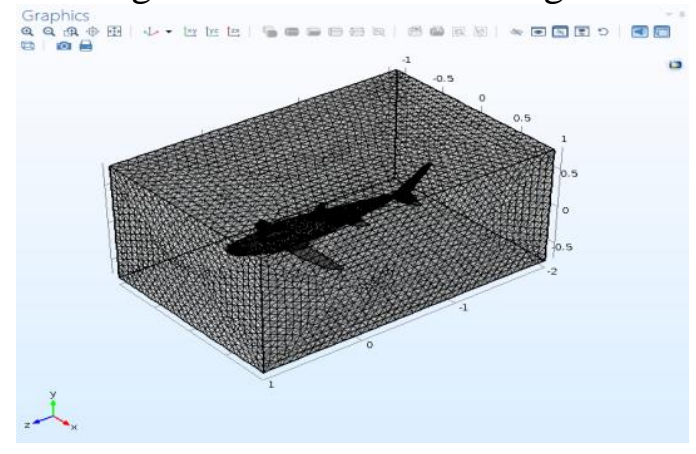

Fig.2. The meshing results of the computational domain 


\section{Numerical Simulation}

First, we selected incompressible fluids as the computational domain fluid properties, according to the actual living environment of prionace glauca, and neglected the effects of heat exchange. In this study, the prionace glauca was immobilized and the flow velocity was increasing. The flow direction was set to the axis $\mathrm{Y}$ of the fish body, and the inlet velocity $V$ was $3 \mathrm{~s}$, which was used to analyze the interaction between the pectoral fins and the flow. And pectoral fins were made by rubber material, elastic modulus is $7.84 \mathrm{MPa}$, density is 1000 , Poisson's ratio is 0.47 .

$$
V=\frac{U t^{2}}{\sqrt{t^{4}-0.06 t^{2}+0.0016}}
$$

where $U$ is the average inlet velocity, $U=1 \mathrm{~m} / \mathrm{s}, t$ is the time. Finally, we carried out post-processing of the simulation results, and computed the resistance changes of prionace glauca under the impact of water flow. Research shown that prionace glauca pectoral fins had obvious deformation in the process of fluid-solid coupling, as shown in Figure 3.

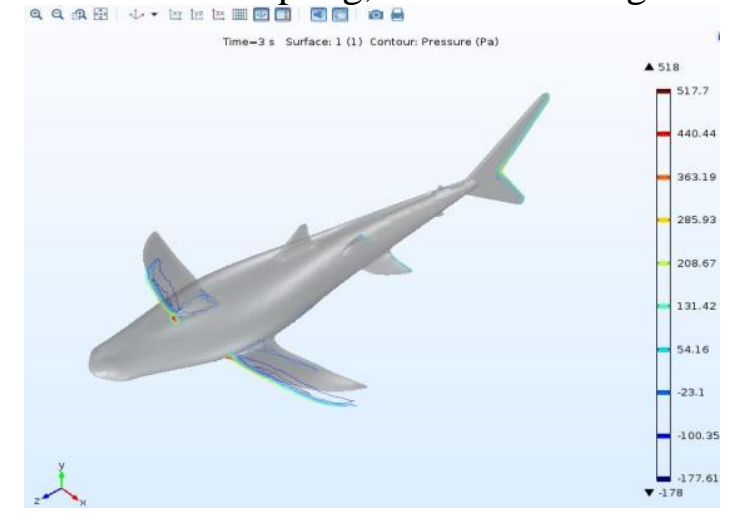

Fig.3. The deformation of prionace glauca pectoral fins in the fluid-solid couplingprocess

\section{COMSOL secondary development}

\section{Setting the App Interface}

COMSOL Multiphysics 5.0 and above version includes the developer of App, which helps to create professional App for everyone from the COMSOL Multiphysics model, and assist engineers to share their models with a wider audience. The App was created after developing the model in COMSOL Multiphysics. The research had assigned the material properties of the prionace glauca pectoral fins, applied the physical field boundary conditions, added the study steps, and customized the drawings of results. The developer of App is fully integrated in the COMSOL Multiphysics, and it has a separate display area in the main screen page tab. Clicking on the developer button of App can enter App developer, as shown in Figure 4.

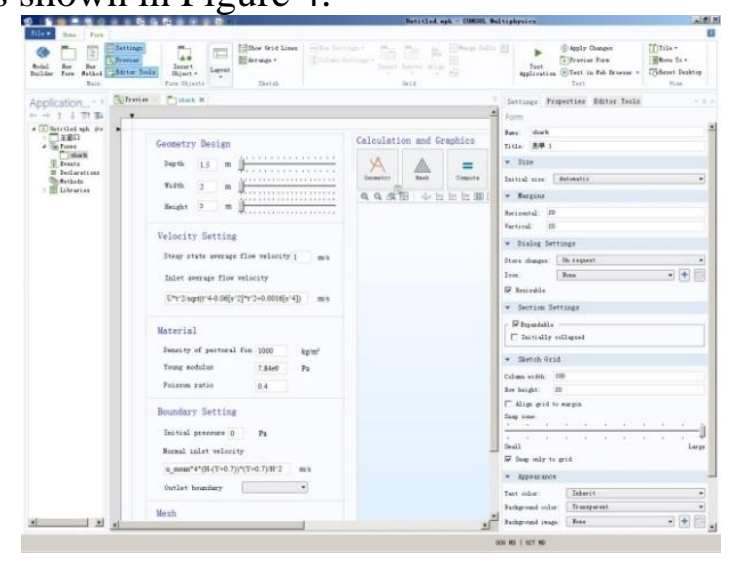

Fig.4.The developer button of App 


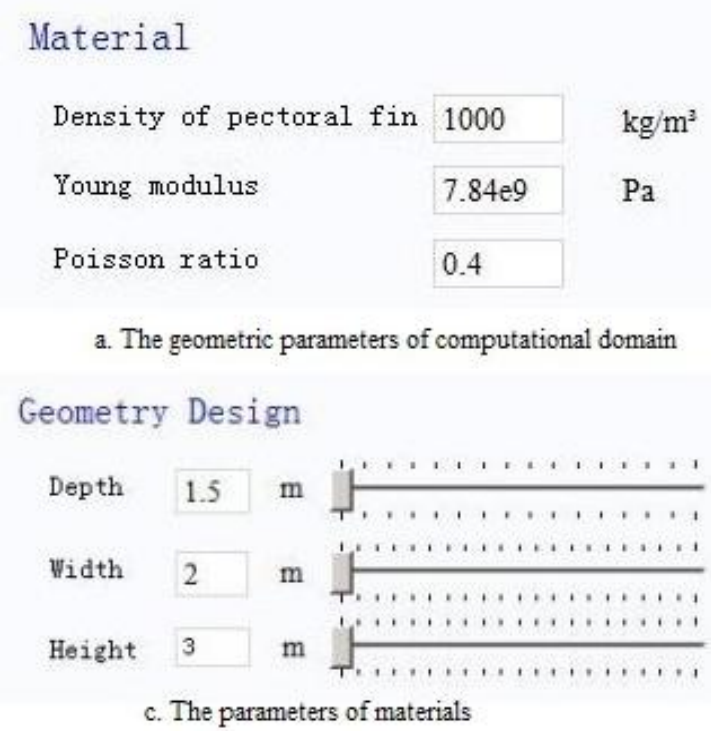

Boundary Setting

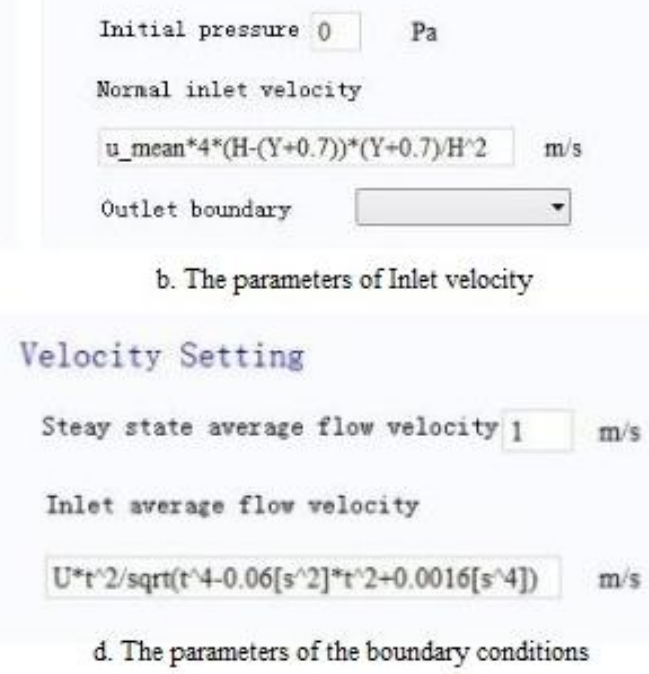

Fig.5.The form interface design of main parameters

App has two developing tools, namely, the form editor and method editor. The research develops App interface by using the form editor, and the main window maintain an orderly structure by referencing the form, and the main window will show the text labels of the input, the results, graphic titles and so on. According to the specific content of this study, the main parameters are extracted and added to the form, as shown in Figure 5.

In the developing process of App, the research integrates the intuitive icons of modelling, grid division, calculation and post-processing, as shown in Figure 6, which facilitate the control of the entire model of numerical calculation and post-processing.

Calculation and Graphics

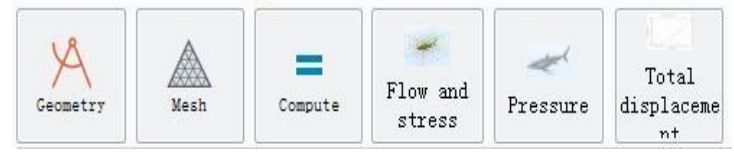

Fig.6. The calculation and post-processing graphics

Through the above editing process, all the forms are integrated in a main window and its layout settings is adjusted and intuitive App interface is tested and formed, as shown in Figure 7. The calculation parameters can be arbitrarily modified in the App, and the whole interface is easy to understand. Even non-professionals can customize their parameters, get the prionace glauca pectoral fins fluid-solid coupling results, and conduct post-processing.

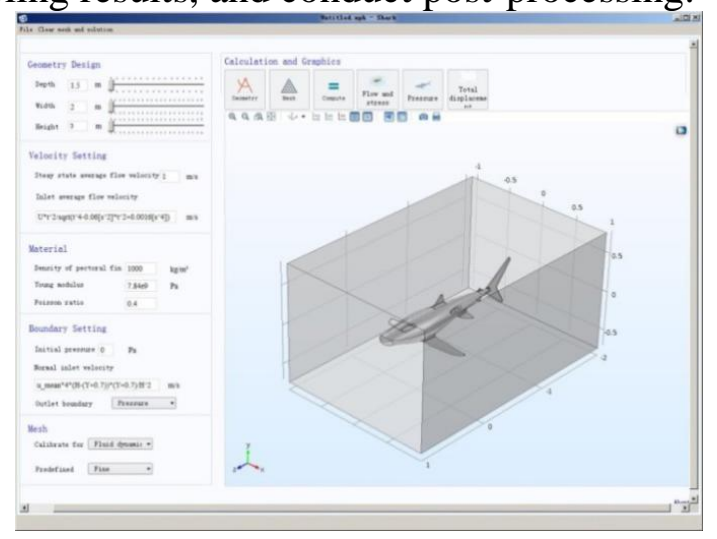

Fig.7.The interface of App 


\section{The Calculation and Post-processing of App}

The fluid-solid coupling numerical calculation parameters of prionace glauca pectoral fins can be directly set through the App interface, and do not need to consider too much of its underlying settings. The App integrates all the buttons of this calculation process, and computes the meshing of the domain, which simplifies the calculation of the domain grid, the control of calculation and post-processing results. Clicking on the fluid and stress buttons, the cloud picture of resistance suffered by prionace glauca fluid-solid interaction can be derived, which visually shows the fluid characteristics and deformation of prionace glauca pectoral fins, as shown in Figure 8. Clicking on the pressure button, the stress distribution of prionace glauca pectoral fins can be displayed. According to Figure 9 shows, the connected parts between prionace glauca pectoral fins and fish body are subjected to greater stress.

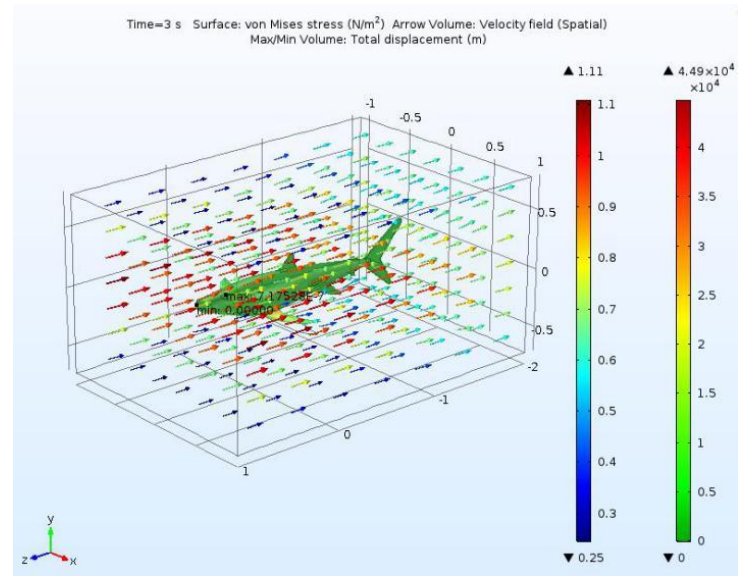

Fig.8.The cloud picture of resistance suffered by prionace glauca

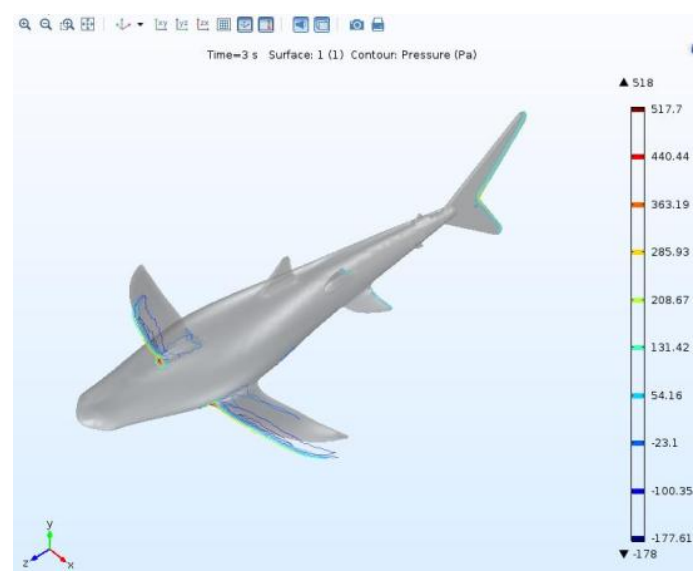

Fig.9.The stress distribution diagram of prionace glauca pectoral fins

The result shows that App does not change the underlying settings of the model, and provides a simple and intuitive user interface that allows the researcher to customize the calculation parameters and post-processing to avoid user input errors, while focusing on the correlation of the output.

\section{Conclusion}

Through the secondary development of COMSOL software, this paper establishes an intuitive and specific user interface, improved modelling capability and post-processing ability for the study of fluid-solid coupling of prionace glauca pectoral fins.

The App developed in this paper makes it easy to modify the parameters of the blue-shark pectoral fin fluid-solid coupling model. Those who do not have the simulation expertise can set their own values to test easily, which dramatically enhances the understanding of fluid-solid coupling simulation calculation, and saves the simulation time. 


\section{Acknowledgement}

This research was financially supported by the National Science Foundation of China(51309150) and Shanghai Municipal Science and technology innovation project (16DZ1205100).

\section{References}

[1] B. D. Bruce, J.D. Stevens, H. Malcolm, Movements and swimming behavior of white sharks (Carcharodon carcharias) in Australian waters. Mar. Biol. 150, 2, 161-172 (2006)

[2] J.M. Donley, R. E. Shadwick, C. A. Sepulvedaet al. Thermal dependence of contractile properties of the aerobic locomotor muscle in the leopard shark and shortfinmako shark. J E Bio,210,7, 1194-203(2007).

[3] Y. Huang, Z.F. Zhou, Pumping test based 3-D evolution inverse analysis for identification of hydrogeological parameters.J H U, 30.6,26-29(2002)

[4] S.Y Wei, K.W Ma, B.B. Li, Searchon Cola and Gas outburstby COMSOL-Multiphysics, C A J E P H, 19, 5 (2010).

[5] H.Z. Li, L.M. H, J. W et al,3D Numerical Simulation of Air Sparging Remediation Process.E S, $33,5,1532-1539(2012)$

[6] R. Wang, Z.Z Shen, X.B Chen,Full coupled analysis of seepage-stress fields for high arch dam based on comsol multiphysics. C J R M\&E, 32, 3197-3204 (2013). 